Case Report

\title{
Vitamin B12 Deficiency and Hemoglobin H Disease Early Misdiagnosed as Thrombotic Thrombocytopenic Purpura: A Series of Unfortunate Events
}

\author{
Panagiotis Andreadis, ${ }^{1}$ Stamatia Theodoridou, ${ }^{2}$ Marily Pasakiotou, ${ }^{3}$ Stergios Arapoglou, ${ }^{4}$ \\ Eleni Gigi, ${ }^{1}$ Evaggelia Vetsiou, ${ }^{1}$ and Efthymia Vlachaki ${ }^{1}$
}

${ }^{1}$ Second Department of Internal Medicine, Aristotle University, Hippokration Hospital, 54642 Thessaloniki, Greece

${ }^{2}$ Thalassemia Unit, Hippokration Hospital, 54642 Thessaloniki, Greece

${ }^{3}$ Intensive Care Unit, Hippokration Hospital, 54642 Thessaloniki, Greece

${ }^{4}$ Fifth Surgical Clinic, Aristotle University, Hippokration Hospital, 54642 Thessaloniki, Greece

Correspondence should be addressed to Panagiotis Andreadis; p.andreadis@hotmail.com

Received 11 July 2015; Revised 9 October 2015; Accepted 15 October 2015

Academic Editor: Uma Dasgupta

Copyright ( 2015 Panagiotis Andreadis et al. This is an open access article distributed under the Creative Commons Attribution License, which permits unrestricted use, distribution, and reproduction in any medium, provided the original work is properly cited.

\begin{abstract}
We herein would like to report an interesting case of a patient who presented with anemia and thrombocytopenia combined with high serum Lactic Dehydrogenase where Thrombotic Thrombocytopenic Purpura was originally considered. As indicated a central venous catheter was inserted in his subclavian vein which led to mediastinal hematoma and finally intubation and Intensive Care Unit (ICU) hospitalization. After further examination patient was finally diagnosed with B12 deficiency in a setting of $\mathrm{H}$ hemoglobinopathy. There have been previous reports where pernicious anemia was originally diagnosed and treated as Thrombotic Thrombocytopenic Purpura but there has been none to our knowledge that was implicated with hemothorax and ICU hospitalization or correlated with thalassemia and we discuss the significance of accurate diagnosis in order to avoid adverse reactions and therapy implications.
\end{abstract}

\section{Introduction}

Thrombotic Thrombocytopenic Purpura (TTP) is a rare lifethreatening blood disorder that is characterized by a pentad of clinical findings including microangiopathic hemolytic anemia (MAHA), thrombocytopenia, fever, renal dysfunction, and neurologic manifestations $[1,2]$. Most of the time, clinical doctors suspect TTP in cases where patients present with thrombocytopenia, elevated Lactic Dehydrogenase (LDH), and schistocytes in peripheral blood smear examination though it is worth mentioning that schistocytes may be absent at the beginning of the disease. Upon serious suspicion of TTP, therapy should be initiated with exchange plasma transfusion [3] that requires insertion of central venous catheter (CVC) [4].

\section{Case Report}

A 58-year-old male patient presented to the Emergency Department complaining of five-day history of worsening fatigue and deteriorating weakness. His medical history was unremarkable. From the clinical examination he was in relative good condition, except presenting with pallor. His vitals were normal and further clinical examination revealed hepatomegaly and splenomegaly (both $5 \mathrm{~cm}$ under the costal arch). Laboratory workup (Table 1) was noteworthy: severe normocytic normochromic anemia with a hematocrit $(\mathrm{Ht})$ of $11 \%$ and hemoglobin $(\mathrm{Hb})$ of $3.8 \mathrm{gr} / \mathrm{dL}$, Mean Corpuscular Volume (MCV) $84.5 \mathrm{fL}$, and increased Red Blood Cell Distribution Width (RDW) 20\%. Platelets (PLTs) count was $130000 / \mathrm{mL}$ while white blood cells (WBCs) count was 
TABLE 1: Patient's blood results: (1) during admission, (2) after the transfusion of 4 packed RBCs units, (3) after the CVC insertion, hemothorax and intubation, (4) at discharge after having received therapy for B12 deficiency, (5) and finally during reevaluation as an outpatient.

\begin{tabular}{|c|c|c|c|c|c|}
\hline & (1) Admission & (2) After transfusion & (3) After CVC placement & (4) Discharge & (5) Reevaluation \\
\hline Hematocrit (\%) & 10.8 & 23.17 & 18.8 & 28.8 & 35.2 \\
\hline Hemoglobin (gr/dL) & 3.1 & 7.99 & 6.36 & 9.2 & 10.9 \\
\hline Red Blood Cells $\left(10^{6} / \mu \mathrm{L}\right)$ & 1.3 & 2.74 & 2.29 & 3.54 & 5.06 \\
\hline $\begin{array}{l}\text { Mean Corpuscular Volume (MCV) } \\
\text { (fL) }\end{array}$ & 84.5 & 82.31 & 85.1 & 81.3 & 69.6 \\
\hline $\begin{array}{l}\text { Mean corpuscular hemoglobin } \\
(\mathrm{MCH})(\mathrm{pg})\end{array}$ & 29.1 & 28.24 & 29.3 & 25.9 & 21.5 \\
\hline $\begin{array}{l}\text { Mean corpuscular hemoglobin } \\
\text { concentration }(\mathrm{MCHC})(\mathrm{g} / \mathrm{dL})\end{array}$ & 33.1 & 34.3 & 33.8 & 31.9 & 30.9 \\
\hline Reticulocytes (\%) & 0.1 & & & & 3 \\
\hline White blood cells $\left(10^{3} / \mu \mathrm{L}\right)$ & 8.50 & 3.9 & 9.5 & 6.34 & 10.81 \\
\hline Neutrophils (\%) & 90.2 & 95.4 & 95.4 & 61 & 66 \\
\hline Lymphocytes (\%) & 8.4 & 3.8 & 3.8 & 16.9 & 24.8 \\
\hline Monocytes (\%) & 0.6 & 0.6 & 0.6 & 8.4 & 5.6 \\
\hline Eosinophils (\%) & 0.1 & 0.06 & 0.06 & 12.9 & 3.7 \\
\hline Platelets $\left(10^{3} / \mu \mathrm{L}\right)$ & 130 & 55 & 25 & 359 & 252 \\
\hline Prothrombin time, PT (Sec) & 12.9 & 12.9 & 15 & 13.2 & 11.2 \\
\hline $\begin{array}{l}\text { International Normalized Ratio } \\
\text { (INR) }\end{array}$ & 1.12 & 1.14 & 1.33 & 1.17 & 0.99 \\
\hline $\begin{array}{l}\text { Activated partial thromboplastin } \\
\text { time (APTT) (sec) }\end{array}$ & 33.6 & 34.8 & 30.9 & 30.6 & 27.8 \\
\hline Fibrinogen $(\mathrm{mg} / \mathrm{dL})$ & 155 & 160 & 139 & 327 & 338.4 \\
\hline Lactic dehydrogenase (LDH) (U/L) & 3700 & 2100 & 1769 & 182 & 252 \\
\hline Coombs Test & Negative & Negative & & & \\
\hline
\end{tabular}

normal. The biochemistry panel had significant findings such as extreme elevation of LDH above $3700 \mathrm{IU} / \mathrm{L}$. Reticulocyte (RC) count was $0.1 \%$. His clotting assay revealed slight elevation of International Normalized Ratio (INR) (1.12) and borderline fibrinogen of $155 \mathrm{mg} / \mathrm{dL}$.

Patient was admitted to our clinic for further investigation and treatment. He was transfused with four Red Blood Cells (RBCs). During series of lab work examination, a drop in the patient's PLT number was observed $(55000 / \mathrm{mL})$. Peripheral blood smear examination during admission revealed no schistocytes. Despite of no such finding at the time, differential diagnosis included TTP, Disseminated Intravascular Coagulation (DIC), and intermediate thalassemia. Since TTP was considered as a possible diagnosis, the insertion of a CVC was mandatory.

After explaining the possible implications of such procedure and written consent, patient underwent surgical placement of central venous catheter on his subclavian vein. The cannulation was achieved by "blind" anatomical landmark method, as in all cases in our hospital. Minutes after the cannulation attempt, patient suddenly presented with perspiration, tachycardia, and pallor. Soon afterwords he lost his consciousness. After regaining his level of consciousness, patient started complaining about pain on his right hemithorax where no respiratory sounds could be auscultated and oxygen saturation dropped rapidly. Patient was intubated due to respiratory failure and hemodynamic instability. He went in cardiopulmonary arrest and was successfully resuscitated and later transferred to the ICU. A thoracostomy tube and Bullau tube were placed and blood output of $2400 \mathrm{~mL}$ was observed. Postprocedure chest radiograph showed large mediastinal hematoma that occupied the right hemithorax. Thoracoscopy revealed multiple bleeding sites. Patient received transfusions with RBCs and Fresh Frozen Plasma (FFP) and his condition stabilized.

Reevaluation of the patient's peripheral blood smear by a specialized hematologist revealed hypersegmented neutrophils, basophilic stippling, anisocytosis and poikilocytosis, reticulocytopenia, and complete absence of schistocytes (Figure 1(a)). Patient underwent bone marrow aspiration and findings included eighty percent erythroblast reaction (erythroid hyperplasia) and few megakaryocytes. Due to high clinical suspicion of megaloblastic anemia, B12 serum levels were ordered and B12 deficiency was confirmed (B12 serum levels $155 \mathrm{pg} / \mathrm{mL}$ after $4 \mathrm{RBC}$ and $3 \mathrm{FFP}$ units transfusion). Patient received treatment with oral folic acid $(5 \mathrm{mg} / \mathrm{d})$ and intramuscular (i.m.) cobalamin $(1000 \mu \mathrm{g} / \mathrm{d})$. Patient's Ht and PLT count were stable and rising. After five days of hospitalization (stay) in the ICU, he was transferred to the surgery department and later on was discharged with instructions for 


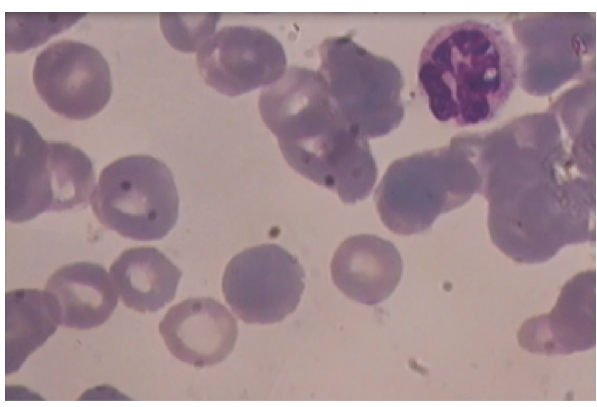

(a)

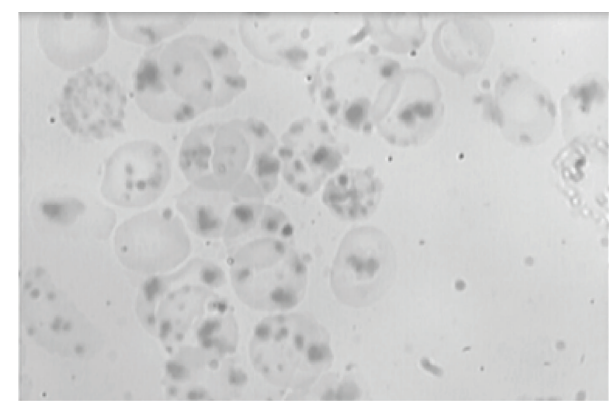

(b)

Figure 1: (a) Peripheral blood smear. A hypersegmented neutrophil is found on the right upper corner of the picture. (b) Peripheral blood smear depicting abundant Heinz bodies in our patient with hemoglobin $\mathrm{H}$ disease.

oral folic acid supplement and i.m. cobalamin once/week for a month and reexamination as an outpatient in our hematology clinic.

During follow-up, patient was feeling well and healthy. Ht was above $35 \%$ and PLT count was between normal ranges (Table 1). Forty-five days after the last blood transfusion, in order to allow apoptosis of the transfused RBCs, peripheral blood smear examination revealed abundant Heinz bodies (Figure 1(b)) and hemoglobin electrophoresis revealed hemoglobin $\mathrm{H}$ disease. Patient underwent endoscopy and gastric mucosal biopsy revealed atrophic gastritis. He is now healthy continuing treatment with vitamin B12 and folic acid.

\section{Discussion}

TTP is a rare blood disorder that pathophysiologically arises from inappropriate clot formation in small blood vessels and results in microangiopathic hemolytic anemia (MAHA) and thrombocytopenia whereas in its course it can present with purpura, neurologic disorders, fever, and renal dysfunction (TTP pentad of clinical findings). The term Thrombotic Microangiopathy (TMA) has been attributed to TTP together with other clinical entities such as hemolytic uremic syndrome (HUS), Disseminated Intravascular Coagulation (DIC), and Antiphospholipid Syndrome (APS) but it may also occur in cases of malignant hypertension, systematic lupus erythematosus, preeclampsia, and others [4]. TTP has been associated with infection, autoimmune diseases, and idiopathic deficiency of ADAMS13 protein. A clinical setting that includes thrombocytopenia, anemia with accompanying elevation in $\mathrm{LDH}$, and indirect bilirubin with negative Coombs Test should give rise to suspicion of TTP. Despite the fact that schistocytes are an expected finding in a setting of MAHA, they might be absent at the beginning of the disease. There have also been reported cases where in the absence of schistocytes TTP was suspected and successfully treated with plasmapheresis $[5,6]$.

Differential diagnosis demands tiering of possible diagnosis according to severity and possibility. TTP is potentially life-threatening and upon clinical suspicion treatment measures should be taken, in this case being plasma exchange transfusion (PEX). Prior to PEX, mortality was nearly $90 \%$.
Furthermore, plasma transfusion and PEX increased survival to 58 and $71 \%$ accordingly [7].

Despite studies about the use of peripheral venous access in PEX $[8,9]$, insertion of a central venous catheter (CVC) is highly indicated and is common practice [10]. Patient must undergo this surgical procedure after written consent and after having been informed about the necessity of the latter and possible implications that include pneumothorax, hemorrhage, deep vein thrombosis, central-line associated infections, and arrhythmias [10, 11]. The placement of CVC may be accomplished using ultrasound guidance or using "blind" anatomical landmark method. There have been metaanalyses that show superiority of the real-time 2D ultrasound assisted-guided CVC placement over the anatomical landmark method [12].

Megaloblastic anemia is a result of defective DNA synthesis and relatively unaffected RNA and cytoplasmic components synthesis. The two latter conditions lead to a delay in cell proliferation and to the formation of "trademark" large RBCs with an arrest in nuclear maturation. Bone marrow findings typically include large erythroblasts (megaloblasts) and hypercellularity [13]. Main morphological feature is macrocytosis which leads to classification in macrocytic anemias and in terms of laboratory findings is correlated with increased MCV. Main causes are nutritional deficiencies such as vitamin B12 (e.g., pernicious anemia) and folic acid, while other causes may include drugs, hypothyroidism, liver disease and alcohol abuse, hemolytic anemia, and myelodysplastic syndromes [14]. Despite the fact that the disease is mostly related with disorders of RBCs, in advanced stages it can affect all cell lines (WBCs, PLTs) and eventually leads to pancytopenia.

Hemoglobin $\mathrm{H}$ disease is a form of $\alpha$-thalassemia syndrome that is mostly observed in areas of the Middle East, Mediterranean, and the tribal belt of India. The disease is characterized by variable degree of $\alpha$-globin chain deficit due to mutations-deletions affecting $\alpha$-globin genes [15]. Due to insufficient production of $\alpha$-globin chain, $\beta$-globin chains create precipitates and form tetramers $\left(\beta_{4}\right)$, known as hemoglobin $\mathrm{H}(\mathrm{Hb} \mathrm{H})$. In many cases, patients remain asymptomatic and transfusion-free. Diagnosis may not be set until the first episode of acute hemolytic crisis $[15,16]$. As 
expected common findings are those of jaundice, indirect hyperbilirubinemia, LDH elevation, and high RC count. Peripheral blood smear may show target cells, microcytosis, hypochromia, and anisopoikilocytosis. Hepatosplenomegaly is a common finding in patients with $\mathrm{Hb} \mathrm{H}$ disease [17]. Vitamin B12 and folic acid deficiencies are very common and may develop in cases of hemoglobinopathies due to increased erythropoiesis. In such cases, patients do not exhibit the expected microcytosis due to lack of factors leading to macrocytosis, though an elevated Red Blood Cell Distribution Width (RDW) is expected. Therefore anemia in $\mathrm{Hb} \mathrm{H}$ disease is primarily a result of hemolysis (due to oxidative stress, infection, or hypersplenism) and secondly a result of ineffective erythropoiesis (due to lack or consumption of necessary cofactors) [15].

\section{Conclusions}

There have been few cases reported to literature where vitamin B12 deficiency has been early misdiagnosed as TTP. Patients with pernicious anemia presented with pseudomicroangiopathic hemolytic anemia that was unsuccessfully treated with plasma exchange transfusions until the establishment of B12 deficiency diagnosis [18-22]. The clinical situation that we describe is unique for two reasons. First of all there have not been previously any reports of pseudo-TMAs in patients with combined B12 deficiency and $\mathrm{Hb} \mathrm{H}$ disease and second of all because early resuscitation measures, that is, central venous catheter insertion, led to severe complications that required ICU hospitalization and intubation. Clinicians should be aware that despite the fact that nutrients deficiency is mostly considered a clinical entity that requires outpatient management and follow-up, patients may also present to the Emergency Department in urgent condition. Doctors should also bear in mind that hemoglobin $\mathrm{H}$ disease and other thalassemias may be an underlying, undiagnosed condition leading to anemia, especially in high endemic areas and where nutrients deficiency may further implicate the course of the disease.

\section{Conflict of Interests}

The authors declare that there is no conflict of interests regarding the publication of this paper.

\section{Acknowledgment}

The paper's subtitle "A Series of Unfortunate Events" was inspired by and taken from the children's novel written by Daniel Handler.

\section{References}

[1] E. Moschcowitz, "An acute febrile pleiochromic anemia with hyaline thrombosis of the terminal arterioles and capillaries: an undescribed disease," Archives of Internal Medicine, vol. 36, no. 1, pp. 89-93, 1952.
[2] J. A. Egan, S. N. Hay, and M. E. Brecher, "Frequency and significance of schistocytes in TTP/HUS patients at the discontinuation of plasma exchange therapy," Journal of Clinical Apheresis, vol. 19, no. 4, pp. 165-167, 2004.

[3] M. Scully and P. Blombery, "Management of thrombotic thrombocytopenic purpura: current perspectives," Journal of Blood Medicine, vol. 2014, article 5, pp. 15-23, 2014.

[4] J. N. George, "How I treat patients with thrombotic thrombocytopenic purpura: 2010," Blood, vol. 116, no. 20, pp. 4060-4069, 2010.

[5] G. A. Rock, K. H. Shumak, N. A. Buskard et al., "Comparison of plasma exchange with plasma infusion in the treatment of thrombotic thrombocytopenic purpura," The New England Journal of Medicine, vol. 325, no. 6, pp. 393-397, 1991.

[6] S. R. Daram, M. Philipneri, N. Puri, and B. Bastani, "Thrombotic thrombocytopenic purpura without schistocytes on the peripheral blood smear," Southern Medical Journal, vol. 98, no. 3, pp. 392-395, 2005.

[7] S. Fava and A. C. Galizia, "Thrombotic thrombocytopenic purpura-like syndrome in the absence of schistocytes," British Journal of Haematology, vol. 89, no. 3, pp. 643-644, 1995.

[8] J. H. Noseworthy, K. H. Shumak, and M. K. Vandervoort, "Long-term use of antecubital veins for plasma exchange. The Canadian Cooperative Multiple Sclerosis Study Group," Transfusion, vol. 29, no. 7, pp. 610-613, 1989.

[9] J. L. Winters, "Plasma exchange: concepts, mechanisms, and an overview of the American Society for Apheresis guidelines," Hematology, vol. 2012, no. 1, pp. 7-12, 2012.

[10] L. Bishop, L. Dougherty, A. Bodenham et al., "Guidelines on the insertion and management of central venous access devices in adults," International Journal of Laboratory Hematology, vol. 29, no. 4, pp. 261-278, 2007.

[11] D. Hind, N. Calvert, R. McWilliams et al., "Ultrasonic locating devices for central venous cannulation: meta-analysis," British Medical Journal, vol. 327, no. 7411, pp. 361-364, 2003.

[12] P. Brass, M. Hellmich, L. Kolodiej, G. Schick, and A. F. Smith, "Ultrasound guidance versus anatomical landmarks for internal jugular vein catheterization," Cochrane Database of Systematic Reviews, 2015.

[13] F. Aslinia, J. J. Mazza, and S. H. Yale, "Megaloblastic anemia and other causes of macrocytosis," Clinical Medicine and Research, vol. 4, no. 3, pp. 236-241, 2006.

[14] V. Hoffbrand and D. Provan, "ABC of clinical haematology. Macrocytic anaemias," British Medical Journal, vol. 314, no. 7078, pp. 430-433, 1997.

[15] D. H. K. Chui, S. Fucharoen, and V. Chan, "Hemoglobin H disease: not necessarily a benign disorder," Blood, vol. 101, no. 3, pp. 791-800, 2003.

[16] S. Fucharoen and V. Viprakasit, "Hb H disease: clinical course and disease modifiers," Hematology, vol. 2009, no. 1, pp. 26-34, 2009.

[17] R. Galanello, B. Aru, C. Dessi et al., "HbH disease in Sardinia: molecular, hematological and clinical aspects," Acta Haematologica, vol. 88, no. 1, pp. 1-6, 1992.

[18] J. K. Routh and S. C. Koenig, "Severe vitamin B12 deficiency mimicking thrombotic thrombocytopenic purpura," Blood, vol. 124, no. 11, p. 1844, 2014.

[19] A. Dimond, J. N. George, and C. Hastings, "Severe vitamin B-12 deficiency in a child mimicking thrombotic thrombocytopenic purpura," Pediatric Blood and Cancer, vol. 52, no. 3, pp. 420-422, 2009. 
[20] K. Tadakamalla Ashvin, K. Talluri Siva, and B. Siddesh, "Pseudo-thrombotic thrombocytopenic purpura: a rare presentation of pernicious anemia," North American Journal of Medical Sciences, vol. 3, no. 10, pp. 472-474, 2011.

[21] N. Noël, G. Maigné, G. Tertian et al., "Hemolysis and schistocytosis in the emergency department: consider pseudothrombotic microangiopathy related to vitamin B12 deficiency," QJM, vol. 106, no. 11, Article ID hct142, pp. 1017-1022, 2013.

[22] V. Mishra, R. Harbada, and A. Sharma, "Vitamin B12 and vitamin d deficiencies: an unusual cause of Fever, severe hemolytic anemia and thrombocytopenia," Journal of Family Medicine and Primary Care, vol. 4, no. 1, pp. 145-148, 2015. 


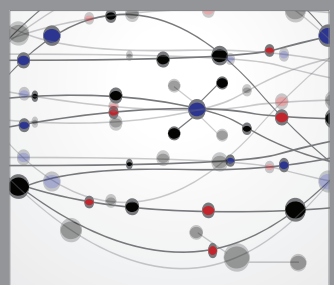

The Scientific World Journal
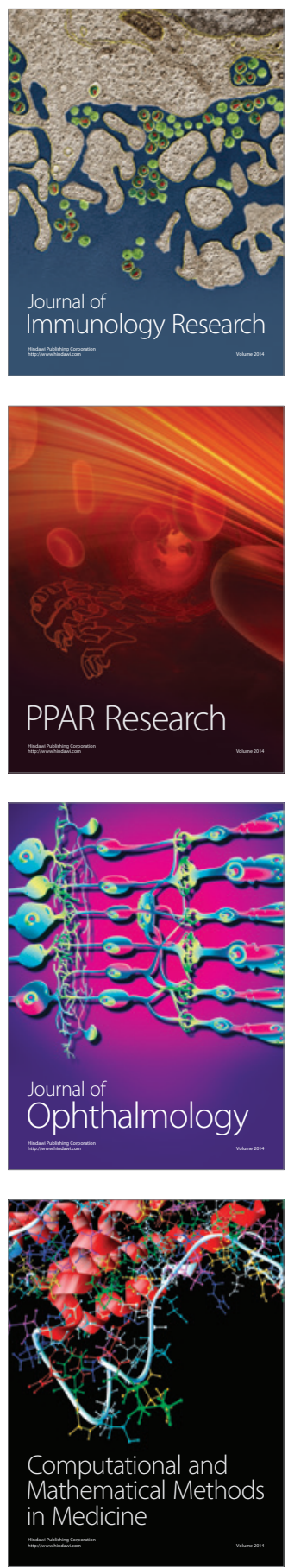

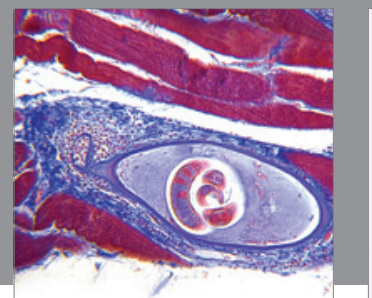

Gastroenterology

Research and Practice
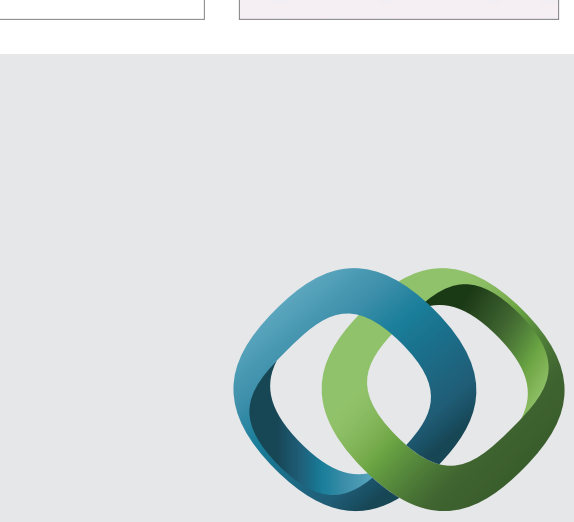

\section{Hindawi}

Submit your manuscripts at

http://www.hindawi.com
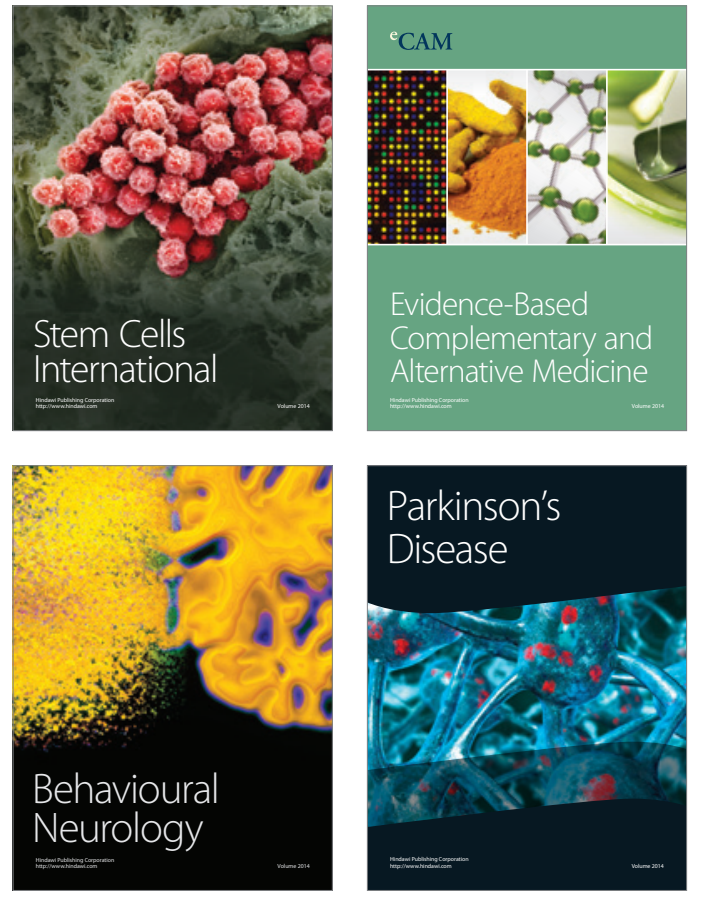
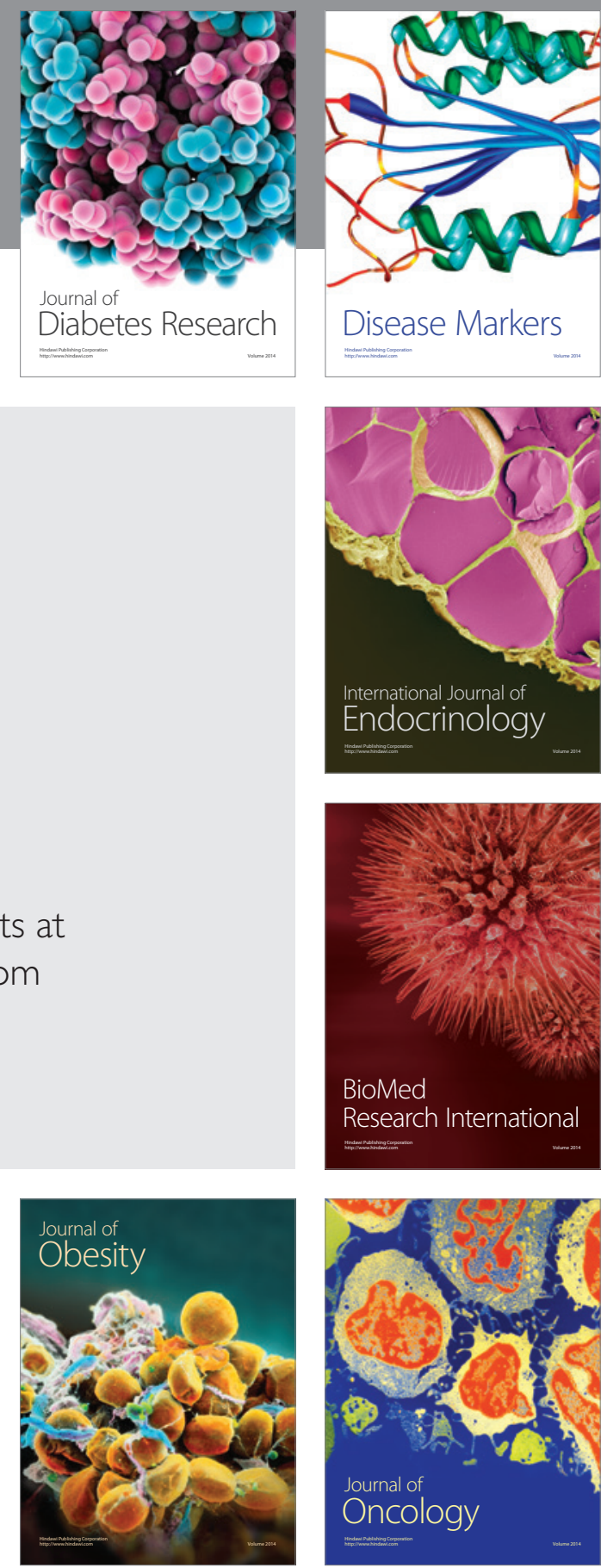

Disease Markers
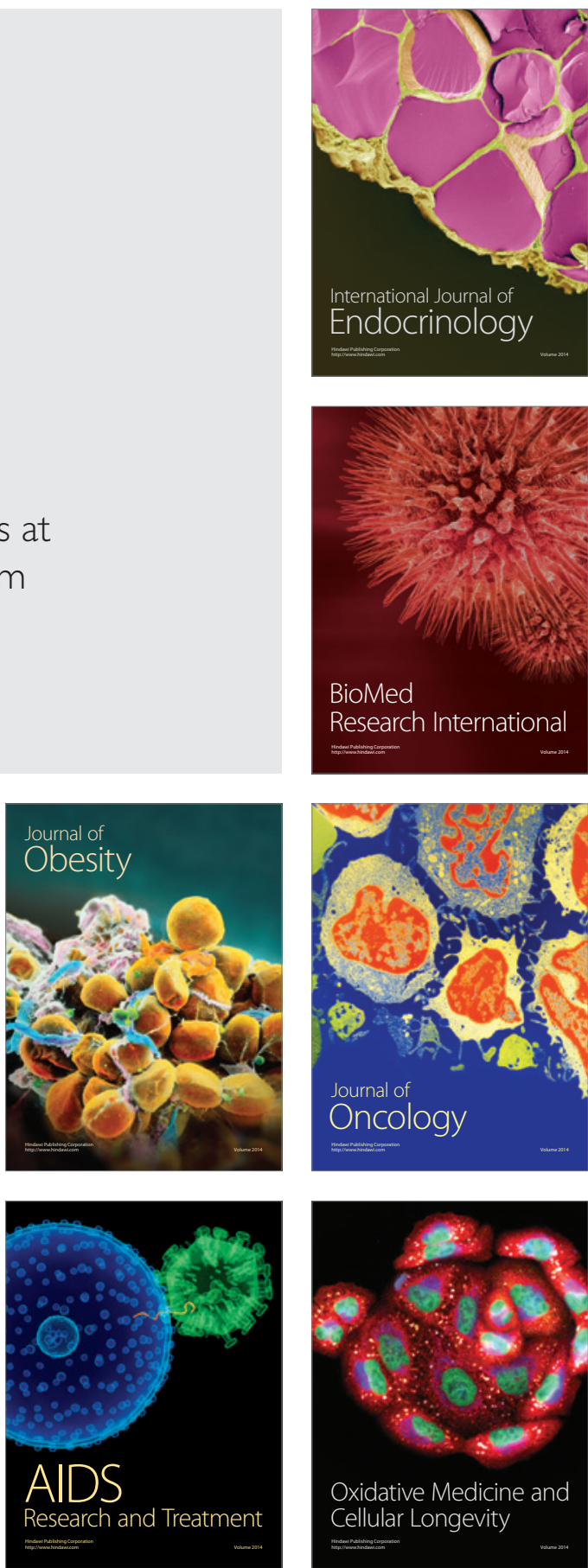